\title{
THE PECULIAR EXTINCTION LAW OF SN 2014J MEASURED WITH THE HUBBLE SPACE TELESCOPE
}

\author{
R. Amanullah ${ }^{1}$, A. Goobar ${ }^{1}$, J. Johansson ${ }^{1}$, D. P. K. Banerjee ${ }^{2}$, V. Venkataraman ${ }^{2}$, V. Joshi ${ }^{2}$, N. M. AshoK ${ }^{2}$,

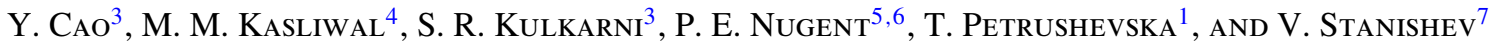 \\ ${ }^{1}$ Oskar Klein Centre, Physics Department, Stockholm University, SE-106 91 Stockholm, Sweden; rahman@ fysik.su.se \\ ${ }^{2}$ Physical Research Laboratory, Ahmedabad 380009, India \\ ${ }^{3}$ Cahill Center for Astrophysics, California Institute of Technology, Pasadena, CA 91125, USA \\ ${ }^{4}$ Observatories of the Carnegie Institution for Science, 813 Santa Barbara Street, Pasadena, CA 91101, USA \\ ${ }^{5}$ Department of Astronomy, University of California Berkeley, B-20 Hearst Field, Annex \# 3411, Berkeley, CA 94720-3411, USA \\ ${ }^{6}$ Computational Cosmology Center, Computational Research Division, Lawrence Berkeley National Laboratory, \\ 1 Cyclotron Road, MS 50B-4206, Berkeley, CA 94720, USA \\ ${ }^{7}$ CENTRA-Centro Multidisciplinar de Astrofísica, Instituto Superior Técnico, Av. Rovisco Pais 1, 1049-001 Lisbon, Portugal \\ Received 2014 April 9; accepted 2014 May 2; published 2014 May 29
}

\begin{abstract}
The wavelength dependence of the extinction of Type Ia SN 2014J in the nearby galaxy M82 has been measured using UV to near-IR photometry obtained with the Hubble Space Telescope, the Nordic Optical Telescope, and the Mount Abu Infrared Telescope. This is the first time that the reddening of an SN Ia is characterized over the full wavelength range of $0.2-2 \mu \mathrm{m}$. A total-to-selective extinction, $R_{V} \geqslant 3.1$, is ruled out with high significance. The best fit at maximum using a Galactic type extinction law yields $R_{V}=1.4 \pm 0.1$. The observed reddening of SN 2014J is also compatible with a power-law extinction, $A_{\lambda} / A_{V}=\left(\lambda / \lambda_{V}\right)^{p}$ as expected from multiple scattering of light, with $p=-2.1 \pm 0.1$. After correcting for differences in reddening, SN 2014J appears to be very similar to SN 2011fe over the 14 broadband filter light curves used in our study.
\end{abstract}

Key words: dust, extinction - galaxies: individual (Messier 82) - supernovae: individual (SN 2014J)

Online-only material: color figures, machine-readable table

\section{INTRODUCTION}

The study of the cosmological expansion history using Type Ia supernovae (SNe Ia), of which SN 2014J is the closest in several decades (Goobar et al. 2014, hereafter G14), has revolutionized our picture of the universe. The discovery of the accelerating universe (Riess et al. 1998; Perlmutter et al. 1999) has led to one of the biggest scientific challenges of our time: probing the nature of dark energy through more accurate measurements of cosmological distances and the growth of structure in the universe. SNe Ia remain among the best tools to measure distances and as the sample grows both in number and redshift range, special attention is required to address systematic effects. One important source of uncertainty is the effect of dimming by dust. Despite considerable effort, it remains unclear why the color-brightness relation for $\mathrm{SNe}$ Ia from cosmological fits is significantly different from, e.g., dimming by interstellar dust with an average $R_{V}=A_{V} / E(B-V)=3.1$. In the most recent compilation by Betoule et al. (2014), 740 low- and high-z SNe Ia were used to build a Hubble diagram using the SALT2 lightcurve fitter (Guy et al. 2007). Their analysis yields $\beta=3.101 \pm 0.075$, which corresponds to $R_{V} \sim 2$, although the assumed color law in SALT2 differs from the standard Milky-Way-type extinction law (Cardelli et al. 1989).

Several cases of $R_{V} \lesssim 2$ have been found in studies of color excesses of local, well-measured, SNe Ia (e.g., Krisciunas et al. 2006; Elias-Rosa et al. 2006, 2008; Nobili \& Goobar 2008; Folatelli et al. 2010). A low value of $R_{V}$ corresponds to a steeper wavelength dependence of the extinction, especially for shorter wavelengths. In general terms, this reflects the distribution of dust grain sizes where a low $R_{V}$ implies that the light encounters mainly small dust grains. Wang (2005) and Goobar (2008) suggest an alternative explanation that non-standard reddening of SNe Ia could originate from multiple scattering of light, e.g., due to a dusty circumstellar medium, a scenario that has been inferred for a few SNe Ia (Patat et al. 2007; Blondin et al. 2009; Dilday et al. 2012).

A tell-tale signature of reddening through multiple scattering is a power-law dependence for reddening (Goobar 2008), possibly also accompanied by a perturbation of the lightcurve shapes (Amanullah \& Goobar 2011) and IR emission from heated dust regions (Johansson et al. 2013).

SN 2014J in the nearby galaxy M82 offers a unique opportunity to study the reddening of a spectroscopically normal (G14; Marion et al. 2014) SN Ia, over an unusually wide wavelength range. Hubble Space Telescope (HST) observations allow us to perform a unique study of color excess in the optical and near$\mathrm{UV}$, where the difference between the extinction models is the largest. Our data set is complemented by UBVRi observations from the Nordic Optical Telescope (NOT) and JHKs from the Mount Abu Observatory.

\section{OBSERVATIONS AND DATA}

\section{1. $H S T / W F C 3$}

We obtained observations (Program DD-13621; PI: Goobar) of SN 2014J with $H S T$ in the four $U V$ broadband filters $F 218 W$, $F 225 W, F 275 W$, and $F 336 W$ for seven epochs using a total of seven HST orbits during Cycle 21. In addition to this we also obtained optical broad-, medium-, and narrowband photometry in filters $F 467 M, F 631 N$, and $F 845 M$ for visits $(1,3)$ and optical broadband photometry using $F 438 W, F 555 W$, and $F 814 W$ for the remaining five visits. All observations were obtained with the Wide-Field Camera-3 (WFC3) using the UVIS aperture UVIS2-C512C-SUB.

The data were reduced using the standard reduction pipeline and calibrated through CALWF3 as integrated into the HST archive. The flat-fielded images were corrected for charge 
Table 1

The Measured Photometry of SN 2014J from HST/WFC3, NOT/ALFOSC, and the Mount Abu Infrared Telescope

\begin{tabular}{|c|c|c|c|c|c|c|c|c|}
\hline $\begin{array}{l}\text { MJD } \\
(1)\end{array}$ & $\begin{array}{l}\text { Phase } \\
\text { (2) }\end{array}$ & $\begin{array}{l}\text { Filter } \\
(3)\end{array}$ & $\begin{array}{c}\text { Mag } \\
(4)\end{array}$ & $\begin{array}{l}A_{X} \\
(5)\end{array}$ & $\begin{array}{c}\text { Match } \\
(6)\end{array}$ & $\begin{array}{c}V \\
(7)\end{array}$ & $\begin{array}{l}A_{V} \\
(8)\end{array}$ & $\begin{array}{c}2011 \mathrm{fe} \\
(9)\end{array}$ \\
\hline 56688.8 & -0.2 & $F 218 W$ & $18.03(0.01)$ & 0.20 & $\mathrm{M}$ & $10.68(0.02)$ & 0.15 & -3.13 \\
\hline 56692.1 & 2.9 & $F 218 W$ & $18.03(0.01)$ & 0.20 & M & $10.67(0.02)$ & 0.15 & -3.14 \\
\hline 56697.0 & 7.3 & $F 218 W$ & $18.35(0.02)$ & 0.20 & $\mathrm{D}$ & $10.81(0.02)$ & 0.15 & -3.53 \\
\hline 56702.9 & 12.7 & $F 218 W$ & $18.88(0.01)$ & 0.19 & M & $11.02(0.02)$ & 0.15 & -4.00 \\
\hline 56713.7 & 22.6 & $F 218 W$ & $19.85(0.03)$ & 0.17 & M & $11.55(0.02)$ & 0.15 & -4.43 \\
\hline
\end{tabular}

Notes. All magnitudes are in the natural Vega system. The rest-frame magnitude can be obtained from Columns 4-5, where Columns 5 and 8 are the Galactic extinctions for the two bands, respectively. Column 2 shows the effective, lightcurve-widthcorrected phase, while Column 6 specifies whether the $V$ magnitude was measured for the same epoch (D) or if it was calculated using the SNooPy model (M). In the latter case the mean error of the data used for the fit was adopted as the uncertainty of the magnitude. The corresponding synthesized color of SN $2011 \mathrm{fe}$ is shown in Column 9.

(This table is available in its entirety in a machine-readable form in the online journal. A portion is shown here for guidance regarding its form and content.)

transfer inefficiencies at the pixel level ${ }^{8}$ and photometry was carried out on the individual images following the guideline from the WFC3 Data Handbook. The individual flat-fielded images were corrected by multiplying with the pixel area map ${ }^{9}$ following Section 7 of the WFC3 Data Handbook. The SN flux could be measured on all images using an aperture with radius 0 .'2. Host contamination is negligible at the $\mathrm{SN}$ position and the statistical uncertainties were estimated assuming Poisson noise of the signal together with the readout noise. The resulting photometry is presented in Table 1.

\subsection{Optical and Near-IR Data}

The UBVRi data were obtained with the NOT (Program 48004; PI: Amanullah). The data were reduced with standard IRAF routines, using the QUBA pipeline (see Valenti et al. 2011, for details). The magnitudes are measured with a point-spread function-fitting technique (using daophot) and calibrated to the Landolt system.

Near-IR (NIR) observations in the Mauna Kea Observatory $J H K s$ filters were carried out with the Mount Abu $1.2 \mathrm{~m}$ Infrared Telescope. Aperture photometry of the sky-subtracted frames was done using IRAF. The nearby star Two Micron All Sky Survey (2MASS) J09553494+6938552, which registers simultaneously with SN 2014J in the same field, was used for calibration. Results were cross-checked with other 2MASS stars in the field and found to agree within 5\%. We adopt this as a systematic uncertainty on the NIR photometry.

All light curves ${ }^{10}$ are presented in Table 1 and Figure 1 where we also show a fitted model to the $V$ band using SNooPy (Burns et al. 2011).

\section{COLOR EXCESS}

\subsection{Intrinsic SN Ia Colors}

In order to study the reddening of SN 2014J, the colors for a pristine, unreddened, SN Ia must be known. Further, we need a color template that includes both the wavelengths and epochs covered by the observations presented in this work.

As described in G14, the early spectral evolution of SN 2014J and SN 2011fe in the nearby spiral galaxy M 101 is remarkably

\footnotetext{
8 J. Anderson 2013, private communication.

9 http://www.stsci.edu/hst/wfc3/pam/pixel_area_maps

10 All tables and figures are available at http://www.fysik.su.se/ rahman/ SN2014J/
}

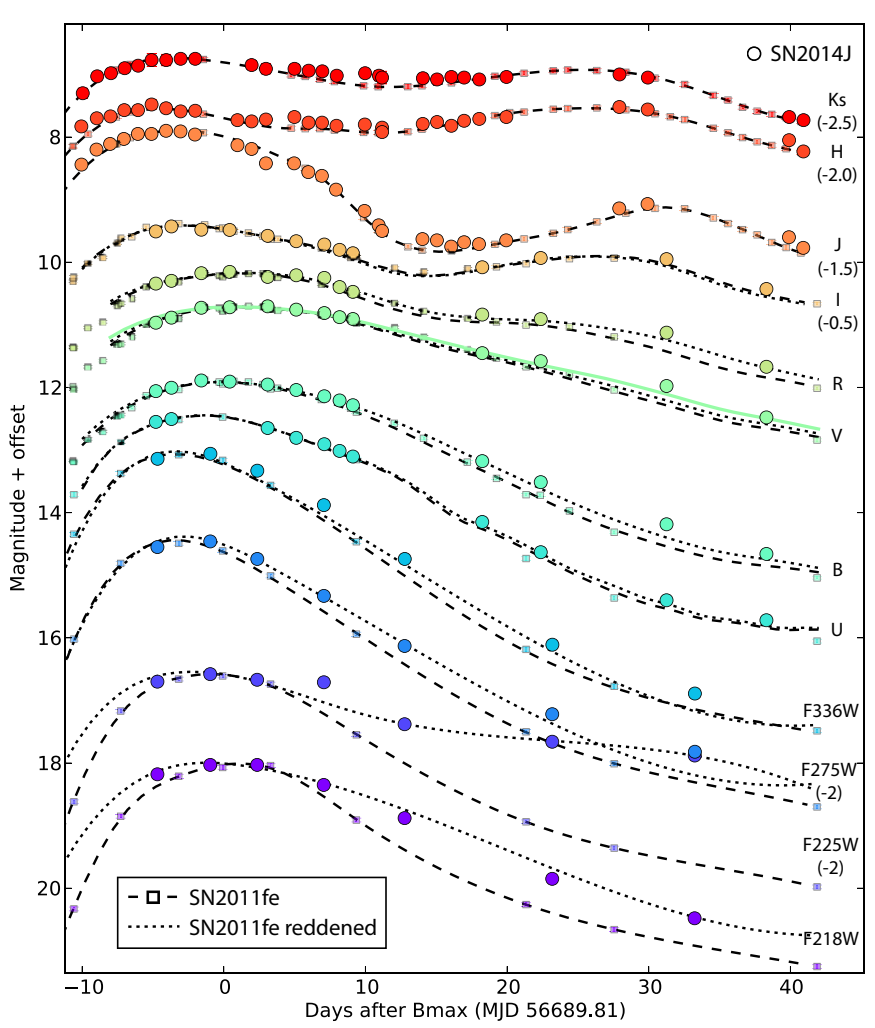

Figure 1. Light curves for all passbands used in this analysis. For the $V$ band we also overplot (solid green line) the fitted model from SNooPy. The black lines are fits to the synthetic photometry of the SN 2011fe spectra (dashed) and the spectra reddened with the best fit FTZ model to SN 2014J.

(A color version of this figure is available in the online journal.)

similar. The only difference being that SN 2014J shows overall higher photospheric velocities. SN 2011fe has been observed over a broad wavelength range from the UV (Brown et al. 2012; Mazzali et al. 2014), through the optical (e.g., Pereira et al. 2013), to the NIR (Matheson et al. 2012; Hsiao et al. 2013). The similarity to SN 2014J together with the low Galactic and host galaxy reddening, $E(B-V)_{\mathrm{MW}}=0.011 \pm 0.002$ and $E(B-V)_{\text {host }}=0.014 \pm 0.002$ mag (Patat et al. 2013), makes SN 2011fe the best template we can derive of the unreddened spectral energy distribution (SED) of SN 2014J.

We use the spectral series from Mazzali et al. (2014) and Pereira et al. (2013), corrected for Galactic extinction using 
Fitzpatrick (1999, FTZ hereafter) with $R_{V}=3.1$, to compute synthetic colors between in the WFC3 and NOT bands in which SN 2014J was observed. The effective HST filters were obtained from SYNPHOT/STSDAS, while we used modified versions of the public effective NOT filters. We further use the SN 2011fe light curves from Matheson et al. (2012) as an unreddened NIR template of SN 2014J. All light curves are shown in Figure 1 (dashed curves) where they have been shifted to overlap with the corresponding SN 2014J photometry at maximum. Smoothed splines are fitted using SNooPy to each individual band to create a pristine lightcurve template.

As seen in Figure 1, the NIR light curves of SN 2011fe provide an excellent description of the corresponding bands of SN2014J, while this is not the case for the bluer bands. At these wavelengths SN2011fe appears to both rise and fall faster than SN2014J, and the difference between the two objects increases with shorter wavelengths. As will be argued in Section 4 this is partially an effect that stems from the fact that broadband observations of SN 2014J are effectively probing longer wavelengths than the corresponding data of SN $2011 \mathrm{fe}$ due to the significant extinction. Taking this effect into account leads to the dotted lines for the reddened SED of SN $2011 \mathrm{fe}$ in Figure 1.

The spectral series will also be used in the analysis to calculate the expected extinction in each passband for a given extinction law. The Mazzali et al. (2014) data set extend out to $\sim 2 \mu \mathrm{m}$ until phase +9 . Since this does not cover the entire phase range of our study we extended this spectral series using the template from Hsiao et al. $(2007,2013)$ for phases past +9 .

In order to compare SN 2014J with SN 2011fe, we also need an estimate of the uncertainty within which we would expect the broadband colors of two SNe Ia to agree in the absence of extinction. Folatelli et al. (2010) studied the intrinsic optical and NIR colors of SNe Ia close to lightcurve maximum, and found dispersions in the range 0.06-0.14 mag, after correcting for the lightcurve shape. We conservatively adopt a dispersion of 0.15 mag (the worst case above) for all colors that only include the optical and NIR bands.

Further, Milne et al. (2010) presented an extensive study of the $U V-V$ dispersion based on observations of $12 \mathrm{SNe}$ Ia with the Swift satellite. For their low-extinction $(E(B-V)<0.2)$ sample they derive dispersions of 0.1 and 0.25 mag between -12 and +12 days relative to the $B$-band maximum for the $u v w 1-v$ and $u v w 2-v$ colors, respectively. We adopt a dispersion of $0.35 \mathrm{mag}$ for the colors that involve $F 218 \mathrm{~W}$ and $F 225 W$ and $0.25 \mathrm{mag}$ for $F 275 W$. For the $V-F 336 W$ dispersion we adopt the same value as the optical range, i.e., $0.15 \mathrm{mag}$. Since UV observations of SNe Ia are scarce, it is difficult to fully assess the differences among SNe at the shortest wavelengths considered here. Foley \& Kirshner (2013) argued that although SN 2011by was a spectral "twin" to SN $2011 \mathrm{fe}$ in the optical, it exhibited a different behavior in the near-UV. We have therefore checked how our estimate of the color excess of SN 2014J would differ under the assumption that it is a better match to SN 2011by instead of SN 2011fe. The offsets at the lightcurve maximum are $\Delta E(V-F 225 W)=0.35 \mathrm{mag}$ and $\Delta E(V-F 275 W)=0.03$ mag, i.e., compatible with our estimate of the intrinsic color scatter.

\subsection{Color Excesses of SN 2014J}

In this work we studied the color excesses, $E(V-X)$, of all photometric bands with respect to the $V$ band. For each photometric observation in Table 1 we also list the corresponding $V$ magnitude. If the $\mathrm{SN}$ was observed in both bands within $12 \mathrm{hr}$ we use the observed $V$ for the corresponding epoch, but when this was not the case we use the fitted $V$-band SNooPy model shown in Figure 1 to calculate the color.

For each epoch we also present the calculated Galactic reddening correction. Unlike G14, we use the Galactic extinction toward M82 from Dalcanton et al. (2009). They argue that the estimates from the dust maps of Schlegel et al. (1998) are contaminated by M82 itself, and derived $E(B-V)_{\mathrm{MW}}=0.06$ from the study of neighboring patches.

We also calculate the corresponding color of SN 2011fe, shown in the last column of Table 1, from the light curves described above. The color excess, $E\left(V_{n}-X_{n}\right)$, between the $V$ band and some other band $X$, at an epoch $n$, can then be obtained under the assumption that the two SNe had nearly identical color evolution. Since the differences in $K$ corrections are negligible for the two very nearby $\mathrm{SNe}$, the color excess is calculated as the difference between the $V_{n}-X_{n}$ color, corrected for Galactic extinction, and the corresponding color of SN 2011fe, i.e.,

$$
E\left(V_{n}-X_{n}\right)=\left[\left(V_{n}-A_{V_{n}}\right)-\left(X_{n}-A_{X_{n}}\right)\right]-\left(V_{n}^{11 \mathrm{fe}}-X_{n}^{11 \mathrm{fe}}\right) .
$$

The first term on the right-hand side, the extinction-corrected color of SN 2014J, is plotted in Figure 2 together with the best fit colors derived from the reddened SED, as described in the next section. In Figure 3, $E\left(V_{n}-X_{n}\right)$ is plotted using data around maximum light.

\section{FITTING EXTINCTION LAWS}

An extinction law, $\xi(\lambda ; \bar{p})$, where $\bar{p}$ are free parameters, can be fitted by minimizing

$$
\chi^{2}=\sum_{X} \sum_{n} \frac{\left[\left(E\left(V_{n}-X_{n}\right)-\left(A_{V_{n}}-A_{X_{n}}\right)\right]^{2}\right.}{\sigma_{V_{n} X_{n}}^{2}} .
$$

Here $E\left(V_{n}-X_{n}\right)$ is the measured color excess as described above and $A_{V_{n}}-A_{X_{n}}$, the corresponding model color excess,

$$
A_{X_{n}}=-2.5 \log _{10}\left(\frac{\int \xi(\lambda ; \bar{p}) T_{X}(\lambda) S_{11 \mathrm{fe}}(\lambda ; n) \lambda d \lambda}{\int T_{X}(\lambda) S_{11 \mathrm{fe}}(\lambda ; n) \lambda d \lambda}\right),
$$

can be calculated from the filter transmission, $T_{X}(\lambda)$, and the SED of SN 2011fe, $S_{11 \mathrm{fe}}(\lambda ; n)$, of the given epoch $n$.

The uncertainties, $\sigma_{V_{n} X_{n}}^{2}$, include the measurement errors shown in Table 1 but is dominated by the adopted intrinsic $\mathrm{SN}$ Ia color uncertainties. If a $V$-band measurement is included in constructing two different colors for a given epoch, then the contribution from the $V$ uncertainty is treated as fully correlated.

In this work we ignore both calibration uncertainties (except for NIR) and systematic errors due to, e.g., Galactic extinction correction. The reason is that these will be correlated between epochs and are negligible in comparison to the intrinsic color uncertainty, which we also assume to be fully correlated. This will put equal weights to all colors, independent of the number of data points obtained in each band.

We further assume that the different colors are uncorrelated in our final analysis. We have tried inducing different correlations between the colors, but this had a minor impact on the conclusions from the fits.

We fitted three different extinction laws: an MW-like extinction law as parameterized by Fitzpatrick (1999, FTZ), the SALT2 law in the version used in Betoule et al. (2014), and a power-law parameterization, $A_{\lambda} / A_{v}=\left(\lambda / \lambda_{V}\right)^{p}$, shown to be a 


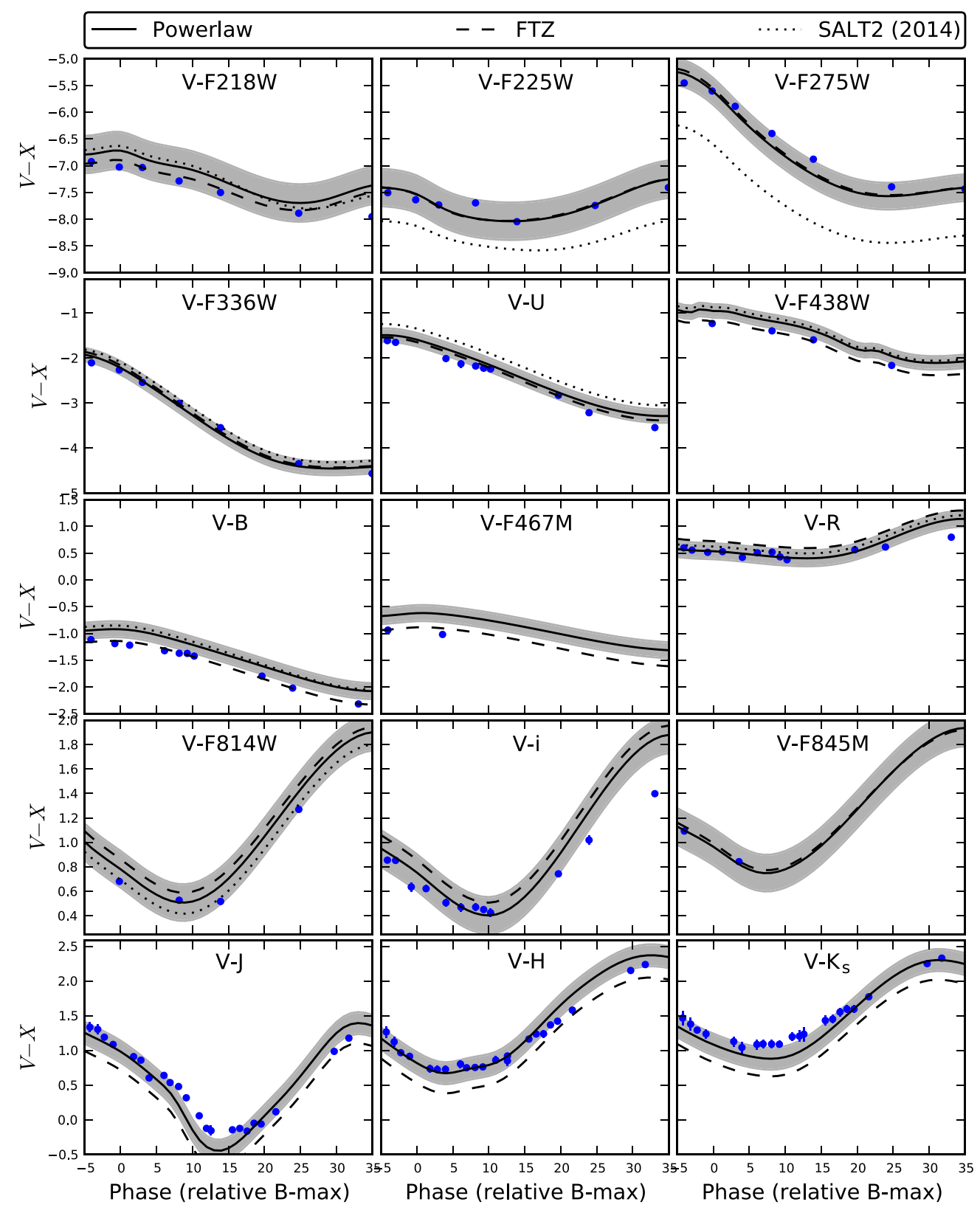

Figure 2. Measured colors (blue points) for the UV, optical, and NIR bands. Also shown are the best fit extinction laws in the $[-5,+35]$ range together with the corresponding predicted colors of SN 2011fe. The gray band shows the adopted intrinsic dispersion of each color plotted with respect to the power-law fit.

(A color version of this figure is available in the online journal.)

good approximation for multiple scattering scenarios (Goobar 2008). Each law was fitted using three different epoch ranges: $[-5,+5]$ days, i.e., around peak brightness, the tail: $[+25,+35]$, and the full epoch range of the HST observations, $[-5,+35]$ days since $B$-band maximum. The FTZ and power law were fitted using all filters, while the SALT2 law is only defined in the wavelength range 2000-7990 A.

The SN colors are compared at the effective phases, computed for both SN 2011fe and SN 2014J by subtracting the date of the $B$-max, 55814.5 and 56689.2, respectively, and dividing by the $i$ lightcurve stretches (from SNooPy), 0.96 and 1.14, respectively. For each law we carry out the fit iteratively to all bands except $F 555 W$ and F631N. The former completely includes our reference band and the latter is too narrow for our assumption of SN 2014J and SN 2011fe being comparable to hold.
We first calculate the Galactic extinction in each band assuming the pristine SED of SN $2011 \mathrm{fe}$. Then, we carry out the fit by minimizing Equation (2). The fitted extinction law is then applied to the SED of SN 2011fe and the Galactic extinction is recalculated before refitting the law. The procedure is repeated until the value of the fitted parameters changes by less than $1 \%$ between iterations. The results of the fits are shown in Table 2, and the best fits to the full range are also shown in Figure 2 while the best fit around maximum is shown in Figure 3. In this figure we also present the best fitted FTZ model with $R_{V}$ fixed to $R_{V}=3.1$, which is clearly excluded by the data. Our best fit FTZ values are $E(B-V)=1.37 \pm 0.03, R_{V}=1.4 \pm 0.1$. We find that our data are also compatible with the power-law model with $A_{V}=1.85 \pm 0.11$ and $p=-2.1 \pm 0.1$. Finally, we conclude that the SALT2 model provides a somewhat poorer fit description with $c=1.06 \pm 0.04$. These findings can 
Table 2

The Best Fitted Parameters for Each Reddening Law to the Broadband Filters

\begin{tabular}{|c|c|c|c|c|c|c|c|c|}
\hline \multirow[t]{2}{*}{ Phase } & \multicolumn{3}{|c|}{ FTZ } & \multicolumn{3}{|c|}{ Power Law: $A_{\lambda}=A_{V}\left(\lambda / \lambda_{V}\right)^{p}$} & \multicolumn{2}{|c|}{ SALT2 (2014) } \\
\hline & $E(B-V)$ & $R_{V}$ & $\chi^{2} /$ dof & $A_{V}$ & $p$ & $\chi^{2} /$ dof & $c$ & $\chi^{2} /$ dof \\
\hline$[-5,+5]$ & $1.37(0.03)$ & $1.4(0.1)$ & 1.1 & $1.85(0.11)$ & $-2.1(0.1)$ & 1.1 & $1.06(0.04)$ & 5.3 \\
\hline$[+25,+35]$ & $1.33(0.04)$ & $1.3(0.1)$ & 1.9 & $1.52(0.11)$ & $-2.4(0.1)$ & 2.2 & $1.10(0.05)$ & 6.9 \\
\hline$[-5,+35]$ & $1.29(0.02)$ & $1.3(0.1)$ & 3.3 & $1.77(0.10)$ & $-2.1(0.1)$ & 2.3 & $1.00(0.01)$ & 5.2 \\
\hline
\end{tabular}

Note. Quoted errors are the uncertainties from the $\chi^{2}$ fit.

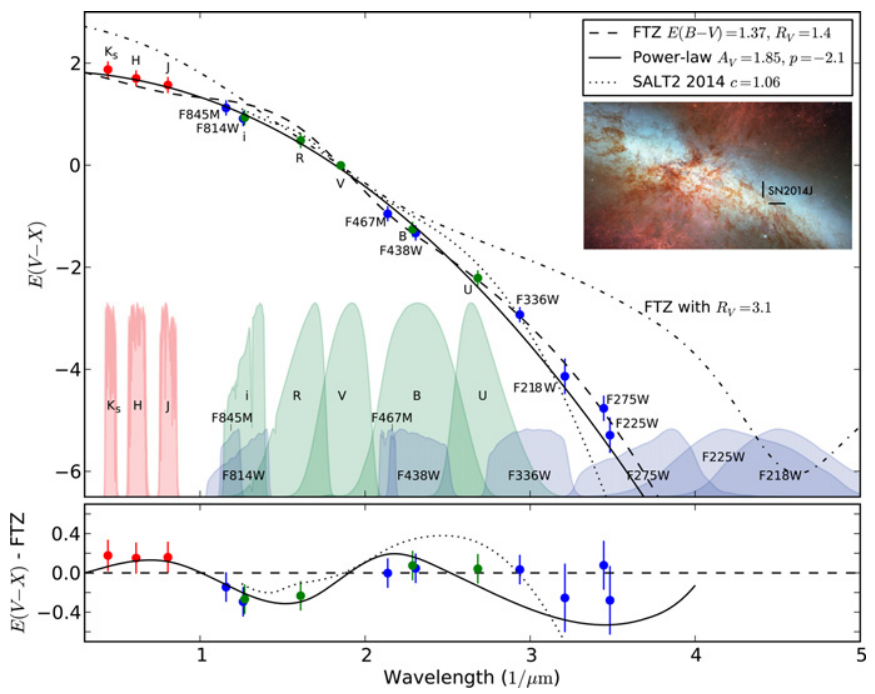

Figure 3. Upper panel shows the average color excesses between -5 and +5 days from the $B$ maximum. Blue, green, and red points are measured with HST, NOT, and the Mount Abu Infrared Telescope, respectively, while the corresponding effective filter transmissions are plotted, in linear scale, at the bottom of the panel. The same data points are plotted as residuals with respect to the best fit FTZ law in the lower panel. For the $U V$ filters the effective wavelengths are significantly redder than the central wavelengths due to the steepness of the reddened SN spectrum.

(A color version of this figure is available in the online journal.)

be compared with the measured global extinction in M82 by Hutton et al. (2014). Unlike our result for the line of sight of SN 2014J, they conclude that FTZ with $R_{V}=3.1$ provides a good description of the colors of the galaxy based on stellar modeling. However, for the dust in the superwind, they too conclude that a power-law relation provides the best fit, albeit with $p=-1.53 \pm 0.17$.

As a consistency check, we recalculate the synthetic light curves of SN 2011fe using the best fitted FTZ law to redden the spectra. The result is plotted in Figure 1 as dotted lines for each band. For the redder bands, these reddened light curves are similar to the original, but for the blue bands, and in particular in the UV, the light curves are significantly broader, and show a similar decline as the observed data of SN 2014J. We take this as yet another confirmation that SN 2011 fe indeed is very similar to SN 2014J and therefore suitable to use as reference for the extinction study presented here. We further conclude (Nugent et al. 2002, and references therein) that the difference in the lightcurve width between the original light curves in the bluer bands mainly stems from the fact that we are probing redder effective wavelengths for SN 2014J than for SN 2011fe when comparing the same passbands.

This also has implications for Figure 3. Here we calculated the weighted average for each color, taking the full covariance into account. However, the color excess is calculated by comparing the magnitudes of a reddened and an unreddened source. The two magnitudes will correspond to different effective wavelengths, and the broader the filter, and the steeper the spectrum, the larger the difference of the two effective wavelengths will become. For Figure 3 we allowed the wavelength to shift, with respect to the average effective wavelength using the FTZ law, until the residuals of the color excess match the weighted average residual from the fit. For the bluest $F 218 \mathrm{~W}$ and $F 225 \mathrm{~W}$, the shift becomes $330 \AA$ and $240 \AA$ respectively. Both of these filters suffer from minor red leaks, e.g., for $F 218 \mathrm{~W} 0.3 \%$ of the light comes from wavelengths redder than $4000 \AA$. On the other hand, an SN Ia at maximum with the reddening of SN 2014J will typically be $\sim 6$ orders of magnitude brighter at $4000 \AA$ compared to the central wavelength of the $F 218 \mathrm{~W}$ filter. The significant shift toward redder wavelengths for these filters is in other words not surprising.

\section{CONCLUSIONS}

We present the results from fitting three extinction laws to observations of SN 2014J in 16 photometric bands spanning the wavelength range $0.2-2 \mu \mathrm{m}$ between phases -5 and +35 days with respect to the $B$ maximum. We find a remarkably consistent picture with the reddening law fits only involving two free parameters. Once reddening is accounted for, the similarity between the multi-color light curves of SN 2014J and SN 2011fe is striking.

We measure an overall steep extinction law with a total-toselective extinction value $R_{V}$ at the maximum of $R_{V}=1.4 \pm 0.1$ for an MW-like extinction law. We also note that the fitted extinction laws are consistent when fitted separately around maximum and using the full phase range.

Although the fits slightly disfavor the empirically derived SALT2 color law for SN Ia, in comparison to an MW-like extinction law as parameterized by FTZ with a low $R_{V}$, conclusions should be drawn cautiously. SALT2 has not been specifically trained for the near-UV region considered here. Also, there is no prediction for the NIR. Intriguingly, the powerlaw extinction proposed by Goobar (2008) as a model for multiple scattering of light provides a good description of the reddening of SN 2014J.

Increasing this sample is crucial to understand the possible diversity in the reddening of $\mathrm{SNe}$ Ia used to measure the expansion history of the universe.

We thank Denise Taylor, Claus Leitherer, and John Mackenty at the Space Telescope Science Institute for advising and assisting us in carrying out this program. R.A. and A.G. acknowledge support from the Swedish Research Council and the Swedish Space Board. M.M.K. acknowledges support from the Hubble Fellowship and Carnegie-Princeton Fellowship. 
Observations were made with the Hubble Space Telescope; the Nordic Optical Telescope, operated by the Nordic Optical Telescope Scientific Association at the Observatorio del Roque de los Muchachos, La Palma, Spain; and the Mount Abu $1.2 \mathrm{~m}$ Infrared Telescope, India. STSDAS is a product of the Space Telescope Science Institute, which is operated by AURA for NASA. V.S. acknowledges support from Fundação para a Ciência e a Tecnologia (Ciência 2008) and grant PTDC/CTEAST/112582/2009.

\section{REFERENCES}

Amanullah, R., \& Goobar, A. 2011, ApJ, 735, 20

Betoule, M., Kessler, R., Guy, J., et al. 2014, A\&A, in press (arXiv:1401.4064)

Blondin, S., Prieto, J. L., Patat, F., et al. 2009, ApJ, 693, 207

Brown, P. J., Dawson, K. S., de Pasquale, M., et al. 2012, ApJ, 753, 22

Burns, C. R., Stritzinger, M., Phillips, M. M., et al. 2011, AJ, 141, 19

Cardelli, J. A., Clayton, G. C., \& Mathis, J. S. 1989, ApJ, 345, 245

Dalcanton, J. J., Williams, B. F., Seth, A. C., et al. 2009, ApJS, 183, 67

Dilday, B., Howell, D. A., Cenko, S. B., et al. 2012, Sci, 337, 942

Elias-Rosa, N., Benetti, S., Cappellaro, E., et al. 2006, MNRAS, 369, 1880

Elias-Rosa, N., Benetti, S., Turatto, M., et al. 2008, MNRAS, 384, 107
Fitzpatrick, E. L. 1999, PASP, 111, 63

Folatelli, G., Phillips, M. M., Burns, C. R., et al. 2010, AJ, 139, 120

Foley, R. J., \& Kirshner, R. P. 2013, ApJL, 769, L1

Goobar, A. 2008, ApJL, 686, L103

Goobar, A., Johansson, J., Amanullah, R., et al. 2014, ApJL, 784, L12

Guy, J., Astier, P., Baumont, S., et al. 2007, A\&A, 466, 11

Hsiao, E. Y., Conley, A., Howell, D. A., et al. 2007, ApJ, 663, 1187

Hsiao, E. Y., Marion, G. H., Phillips, M. M., et al. 2013, ApJ, 766, 72

Hutton, S., Ferreras, I., Wu, K., et al. 2014, MNRAS, 440, 150

Johansson, J., Amanullah, R., \& Goobar, A. 2013, MNRAS, 431, L43

Krisciunas, K., Prieto, J. L., Garnavich, P. M., et al. 2006, AJ, 131, 1639

Marion, G. H., Sand, D. J., Hsiao, E. Y., et al. 2014, ApJ, submitted (arXiv:1405.3970)

Matheson, T., Joyce, R. R., Allen, L. E., et al. 2012, ApJ, 754, 19

Mazzali, P. A., Sullivan, M., Hachinger, S., et al. 2014, MNRAS, 439, 1959

Milne, P. A., Brown, P. J., Roming, P. W. A., et al. 2010, ApJ, 721, 1627

Nobili, S., \& Goobar, A. 2008, A\&A, 487, 19

Nugent, P., Kim, A., \& Perlmutter, S. 2002, PASP, 114, 803

Patat, F., Chandra, P., Chevalier, R., et al. 2007, Sci, 317, 924

Patat, F., Cordiner, M. A., Cox, N. L. J., et al. 2013, A\&A, 549, A62

Pereira, R., Thomas, R. C., Aldering, G., et al. 2013, A\&A, 554, A27

Perlmutter, S., Aldering, G., Goldhaber, G., et al. 1999, ApJ, 517, 565

Riess, A. G., Filippenko, A. V., Challis, P., et al. 1998, AJ, 116, 1009

Schlegel, D. J., Finkbeiner, D. P., \& Davis, M. 1998, ApJ, 500, 525

Valenti, S., Fraser, M., Benetti, S., et al. 2011, MNRAS, 416, 3138

Wang, L. 2005, ApJL, 635, L33 